Children; ${ }^{3}$ Department of Obstetrics and Gynecology, The Prenatal Diagnosis and Medical Genetics Program, Mount Sinai Hospital, University of Toronto, Toronto, ON, Canada

Williams syndrome is a rare genetic neurodevelopmental disorder with a characteristic physical and behavioral phenotype caused by deletion at the 7q11.23. It is usually diagnosed in childhood by clinical evaluation when typical facial features, supravalvular aortic stenosis on echocardiography, hypercalcemia and other neurodevelopmental and behavioral profile may become apparent. Conjugated hyperbilirubinemia, posterior embryotoxon, pulmonary stenosis, vertebral anomalies, renal anomalies and vascular anomalies are typical features of Alagille syndrome, which is caused by mutations in or deletion of the JAG1 gene at 20 p. 12 or rarely the NOTCH 2 gene at $1 \mathrm{p} 12$. There may be some overlap in the clinical features between these syndromes; however, conjugated hyperbilirubinemia, posterior embryotoxon and vertebral anomalies are not features of William syndrome. The typical facial features specific to each of the syndromes usually become apparent with age and pose a challenge in making a diagnosis in the newborn period and especially when the baby is premature. We report a preterm newborn with spectrum of clinical features highly suggestive of Alagille syndrome but array CGH consistent with Williams syndrome. To the best of our knowledge, this very unusual association, has been reported only on three occasions in the past and further extend the phenotype of Williams syndrome.

\section{0 \\ EFFECT OF TRANEXAMIC ACID IN THE MANAGEMENT OF HEMOPHILIA}

doi:10.1136/archdischild-2012-302724.0560

BL Bajracharya. Paediatrics, Kathmandu Medical College Teaching Hospital, Kathmandu, Nepal

Background and Aims Hemophilia is a rare genetic disease. Treatment of hemophilia is a great burden to the patient, family as well as to the nation.

Recombinant factor concentrates, currently available products are viral pathogen-free, although there is debate about the risk of transmission of parvovirus B19 and prion pathogens. Because of this very small risk, recombinant factor is the treatment of choice in hemophilia patients.

In developing country like Nepal the treatment is based on the blood product like Fresh frozen plasma and cryo precipitates. Recombinant therapy is very expensive and not readily available in local market.

Treatment with tranexamic acid has been tried with success in the management of minor bleedings at hemophilia care unit, Kathmandu Medical college Teaching hospital which has reduced the necessity of use of blood product and the cost of treatment.

Aim of this study is to see the effect of tranexamic acid in oral hemorrhage, to reduce the cost of treatment to avoid blood product.

Method Retrospective study.

Results Bleeding stopped in all patient with gum bleeding within few hours of treatment whereas bleeding did not stopped in any patient with tongue injury.

Conclusions Extremely useful in the control of mucous membrane bleeding. Main advantage is inexpensive and no risk of bloodborne viral infections.

\section{For oral presentation}

Disclosure of financial relationships There was no financial support from any manufacturer/supplier of the commercial products related to this work.

\section{PRESENTATION OF RARELY SEEN GASTROINTESTINAL TELANGIECTASIA IN A 4 YEAR OLD CHILD WITH RARE CONDITION GLANZMANN'S THROMBASTHENIA}

doi:10.1136/archdischild-2012-302724.0561

${ }^{1}$ A Silwal, ${ }^{2} \mathrm{M}$ Richards. ${ }^{1}$ Calderdale and Huddersfield NHS Trust, Halifax; ${ }^{2}$ Department of Paediatric Haemato-Oncology, Leeds Teaching Hospitals NHS Trust, Leeds, UK

A 4 year old girl diagnosed with Glanzmann's Thrombasthenia at the age of 5 months had been admitted on previous occasions to hospital with epistaxis.

This encounter describes her presentation with first episode of haematemesis. No focal bleeding source was noted on ENT examination.

Emergency endoscopy showed discrete telangiectasia in stomach. The combination of GI telangiectasia and Glanzmann's Thrombasthenia has been rarely reported.

\section{RADIOLOGICAL EVALUATION OF PEDIATRIC CONGENITAL URINARY TRACT ANOMALIES}

doi:10.1136/archdischild-2012-302724.0562

SS Hwang, SY Park. Radiology, St. Vincent's Hospital, The Catholic University of Korea, Suwon, Republic of Korea

Purpose To illustrate a wide spectrum of congenital anomalies of the urinary tract in children.

Materials and Methods We demonstrate radiological evaluation and its clinical significance of congenital anomalies of the urinary tract in pediatric patients.

Results Demonstrated various pediatric congenital urological anomalies included kidney (renal agenesis, ectopic kidney, multicystic dysplastic kidney, duplication), ureter (primary megaureter, ectopic ureterocele, ectopic insertion of ureter), bladder (anterior bladder diverticulum), and urethra (posterior urethral valve, urethral diverticulum, urethral polyp). We also described its clinical significance.

Conclusion Radiological evaluation including Ultrasonography, CT, and/or MRI is very useful for diagnosis and follow-up of pediatric urologic structural anomalies.

\section{CASE REPORT: TRANSVERSE MYELITIS CAUSED BY ENTEROVIRUS INFECTION}

doi:10.1136/archdischild-2012-302724.0563

BES Araujo, APDC Dalcol, A Vasconcellos, SHS Victal, AC Seabra. Cetipen, Hospital Central da Policia Militar Rio de Janeiro, Rio de Janeiro, Brazil

Background and Aims Enterovirus infections are most common in young children, and acute infection of the CNS occurs at all ages. Meningitis is by far the most common CNS manifestation. Certain enteroviruses (ie, polioviruses, enterovirus 71) preferentially target the motor nuclei and anterior horn cells of the brain and spinal cord, causing acute paresis of cranial and spinal nerves.

Our objective is report an uncommon case of a teenage girl with transverse myelitis caused by enterovirus infection whose obtained good outcome after plasmapheresis treatment.

Methods Report case.

An 11 years old girl with lower limb paresthesia, are flexia, hypotony, evolving with bladder's urine retention, paresis and respiratory effort about 10 days. The liquor exam was positive for enterovirus PCR (polymerase chain raction) and thoraco lumbar NMR revelead transverse myelitis involving C3 and L4 level. 